population growth. As stressed by Korten (Studies in Family Planning, 6, 178-187; 1975), there are few other ideas in human history that have been transformed from taboo to official government policy in little more than a decade. Even so, the picture is not encouraging. The survey of aims and accomplishments with respect to population growth in the 14 largest developing countries (in order, China, India, Indonesia, Brazil, Nigeria, Bangladesh, Pakistan, Mexico, Philippines, Thailand, Turkey, Egypt, Iran, Korea) by Stewart (in Population and the New Biology, Academic Press, New York, 1974) reveals a consistent pattern of targets which, although modest enough, are not being met. India's twothirds cut-back in the 1974 budget for its family planning programme is the sort of economy it can least afford.

Another interesting demographic statistic is the percentage of a population that is classed as urban. This statistic is made a little fuzzy by virtue of the definition of an "urban" locality differing from country to country (see Trends and Prospects in Urban and Rural Populations, 1950-2000, UN, New York, 1975). Nonetheless, this figure shows interesting trends, parti-

cularly when the estimates for 1975 are put beside those for 1950 .

In $1950,29 \%$ of the world population was urban, as against $39 \%$ in 1975. Broken down into geographical groupings, these figures (expressed as percentage urban in 1950 and in 1975 , respectively) are: 23 and 44 for southwest Asia; 16 and 21 for middle south Asia; 13 and 22 for southeast Asia; 17 and 31 for east Asia; 13 and 25 for Africa; 41 and 60 for Latin America; 55 and 67 for Europe; 39 and 61 for the USSR; 64 and 77 for North America; and 65 and 72 for Oceania. Concealed within these aggregated numbers are such dramatic transformations as $28 \%$ urban growing to $63 \%$ over 25 years in Martinique, 11 to $37 \%$ in Zambia, 21 to $50 \%$ in Algeria, and many other examples of a more than $200 \%$ change in the urban fraction, particularly in Africa. Very little of this differential growth in urban populations is the result of planning. The absolute lowest urban fractions in 1975 come from Nepal ( $5 \%$ ), followed by Tanzania (7) and Uganda (8). The most urban large country in both 1975 and 1950 was Australia (with 86 and $80 \%$, respectively), slouch-hat jackeroo image to the contrary.

\title{
Conservation of microbial genetic resources
}

from S. P. Lapage

\begin{abstract}
The Third International Conference of Culture Collections (ICCC III), was held in Bombay, from March 15-19, 1976 and was attended by 290 scientists from 29 countries. It was organised by the World Federation of Culture Collections and local organising committee, under the auspices of UNEP, Unesco, ICRO and the UNEP/Unesco/ICRO panel, IUBS, The University of Bombay and other Indian organisations.
\end{abstract}

ICCC III provided a useful forum for discussion on the role and development of culture collections in developing countries. Emphasis was placed on the preservation of strains for local use and the preservation of those local strains which might otherwise be lost to science; on the relation of culture collections to the UN agencies and to the proposed development of regional microbial resource centres, with associated culture collections that would maintain the relevant strains of microorganisms, (for example suitable strains for nitrogen fixation or food fermentation in the particular region).

Also discussed were the value of national and regional federations of culture collections, the World Data
Center and its relation to other data centres and their services in providing information on the location of the microbial genetic resources held in culture collections. The related problems of standardisation of data handling and of data coding for groups of microorganisms were also considered extensively, and it was thought necessary that the agreement of the relevant international authority for each group of microorganisms concerned (such as the International Committee of Systematic Bacteriology and International Committee on the Taxonomy of Viruses) be obtained before any system of data coding was universally recommended or adopted. The deposit of microorganisms for patent procedure in culture collections and an International Treaty proposed under the sponsorship of the World Intellectual Property Organisation were also discussed, as were the problems of identification in culture collections, and the value of culture collections in many other fields-medical, veterinary, industrial and others.

The technical aspects of preservation were also dealt with. T. W. G. Rowe (Edwards High Vacuum, Crawley, UK) announced some recent work of D. Greiff (Medical College of Wisconsin, Milwaukee) who has shown that ice and DMSO can be simultaneously sublimated under vacuum from a protein solution to leave a water-soluble 'cake', which opens up a prospect for the successful freeze-drying of microorganisms and possibly tissue cultures that do not survive freezing without a cryoprotectant. Rowe and Greiff have also shown the persistence of minute open channels in the heat sealed ends of Pyrex glass ampoules through which air and water vapour may percolate leading to deterioration of the contents. These channels can be closed simply by coating the sealed end with the synthetic rubber, neoprene.

\section{Drug action at the molecular level}

\author{
from S. Neidle
}

\begin{abstract}
A symposium on this topic was held on April 12-13 at the Middlesex Hospital Medical School, London, organised by the Co-ordinating Committee for Symposia on Drug Action, under the auspices of the Biological Council.
\end{abstract}

MOLecular pharmacology as an area of study encompasses a wide range of disciplines, from X-ray crystallography through membrane transport kinetics, to bio-organic chemistry. This diversity of approach was well illustrated at the recent symposium on drug action. Although the contributions covered a number of different problems, it became clear however, that many of these approaches are indeed to a large extent interdependent. Thus, "hard" results from say, kinetic studies concerning a biological receptor and the drugs which bind to it are best obtainable when the receptor itself has an established molecular structure. This point was exemplified by P. J. Goodford (Wellcome Research Laboratory), who described recent attempts to design a drug to fit a known receptor. Haemoglobin, whose three-dimensional structure is well understood, was used as a model. Diphosphoglycerate, a natural inhibitor of oxygen uptake by haemoglobin, fits into a geometrically welldefined binding cleft in the protein. Instead of synthesising series of diphosphoglycerate analogues, Goodford and his colleagues simply decided to manufacture a molecule that would fit exactly into the cleft, and have appropriate groupings to confer hydrogen-bonding donor and acceptor properties. Biological testing of the inhibitor properties of their synthetic substrates have provided results which 